\title{
Real-World Analysis of Treatment Patterns Among Hospitalized Patients with Pulmonary Arterial Hypertension
}

\author{
Adesuwa Ogbomo • Yuen Tsang • Furaha Kariburyo - Wan-Lun Tsai • \\ Sumeet Panjabi
}

Received: July 8, 2021 / Accepted: August 26, 2021 / Published online: October 26, 2021

(C) The Author(s) 2021

\section{ABSTRACT}

Introduction: Hospitalization is an important clinical factor associated with survival and rehospitalization in patients with pulmonary arterial hypertension (PAH). Thus, this study examined treatment patterns before and after hospitalization in the US-specific population.

Methods: Adult PAH patients in the United States were identified using the Optum ${ }^{\circledR}$ Clinformatics ${ }^{\circledR}$ database from January 1, 2014, to June 30,2019 , and were required to have continuous health plan enrollment for at least 6 months prior to the first (index) hospitalization through at least 90 days post-discharge. Baseline patient characteristics were evaluated from 6 months prior to through the index hospitalization. PAH treatment patterns were examined from 30 days pre-index admission (pre-hospitalization) and 90 days post-index hospital discharge (post-hospitalization), and stratified by therapy type: monotherapy,

Supplementary Information The online version contains supplementary material available at https:// doi.org/10.1007/s41030-021-00173-6.

A. Ogbomo $(\bowtie) \cdot$ F. Kariburyo · W.-L. Tsai Real World Evidence, STATinMED Research, Ann Arbor, MI, USA

e-mail: aogbomo@statinmed.com

Y. Tsang $\cdot$ S. Panjabi

Janssen Scientific Affairs, Titusville, NJ, USA double- or triple-combination therapy, or no PAH therapy.

Results: A total of 3116 hospitalized patients with PAH met selection criteria. The mean age and Charlson comorbidity index score were 68.1 years and 5.1, respectively. In the pre- and post-hospitalization periods (all-cause), respectively, patients prescribed monotherapy were most common (from $64.8 \%$ pre- to $51.9 \%$ posthospitalization), followed by patients with no evidence of PAH therapy (from 14.6 to $28.5 \%$ ). Among PAH-related hospitalizations, patients with monotherapy were also most common (from $60.8 \%$ pre- to $49.1 \%$ post-hospitalization), followed by patients with no evidence of PAH therapy (from 10.0 to $22.8 \%$ ). The majority of patients with all-cause hospitalizations $(72.8 \%)$ had no therapy modification; $20.0 \%$ de-escalated therapy (including $15.0 \%$ from monotherapy to no therapy) and $6.1 \%$ escalated therapy (including $2.2 \%$ from no therapy to monotherapy and $3.2 \%$ from monotherapy to double or triple therapy).

Conclusion: Inpatient admissions did not appear to drive changes in PAH therapy management, as monotherapy predominated, and most patients had no therapy modification within 90 days of a hospitalization. These results warrant future research to understand the reasons behind the limited treatment intensification observed and the impact of posthospitalization optimization on clinical and economic outcomes. 
Keywords: Pulmonary arterial hypertension; Hospitalization; Combination therapy

\section{Key Summary Points}

Why carry out this study?

Pulmonary arterial hypertension (PAH) is a rare but incurable disease with considerable morbidity and mortality worldwide and in the USA.

Recent research and clinical guidelines suggest early intensification of PAH therapy with combination therapies.

Hospitalization is an important prognostic indicator for PAH progression, but little is known about treatment intensification coinciding with hospitalization.

\section{What was learned from the study?}

Results showed a majority of patients were prescribed monotherapy both before and after hospitalization, and a majority had no treatment intensification after hospitalization.

There is a need for more research to understand the reasons driving the gap between clinical practice and the evidence and guideline recommendations for initial combination therapies, as well as treatment intensification upon hospitalization.

\section{INTRODUCTION}

Pulmonary arterial hypertension (PAH) is an incurable and progressive disease characterized by sustained idiopathic pulmonary vascular resistance, which eventually leads to right heart failure and premature death $[1,2] \mathrm{PAH}$ is $3-5$ times more common among women than men, and it often remains undiagnosed or misdiagnosed for extended periods [2]. The disease is incapacitating as it progresses, and it has been associated with multimorbidity that may contribute to underdiagnosis [3, 4]. Although rare, with an incidence ranging from 1.5 to 32 cases per million, and prevalence ranging from 12.4 to 268 cases per million between 2003 and 2020 within the USA, Europe, and Asia [5], PAH is responsible for considerable clinical and economic burden [6].

While PAH remains incurable, the treatment landscape has evolved over the past two decades toward considerably increased survival and improved quality of life [7]. This is in part attributable to the introduction of combination therapies that have shown superior efficacy as compared with traditional monotherapy $[8,9]$. Clinical guidelines (European Society of Cardiology [ESC] and the European Respiratory Society [ERS]; CHEST Guideline and Expert Panel Report) for PAH therapy also recommend more timely and aggressive use of combination therapies, in order to utilize agents with mechanisms of action that target different signaling pathways $[1,10]$. These guidelines include specific recommendations for initial combination therapy for patients with severe $\mathrm{PAH}$ (World Health Organization functional class [WHO FC] IV) and certain mild to moderate cases (WHO FC II-III) accompanied by rapid progression or failure to meet prognostic benchmarks in goal-oriented therapy, such as exercise capacity and echocardiographic, laboratory, and hemodynamic variables $[1,10]$. There is some evidence suggesting that treatment patterns have been gradually trending toward wider use of combination therapies [7]. However, more evidence is required to evaluate the traction these guidelines have in current clinical practice.

Hospitalization is an important prognostic indicator of PAH disease progression, as recent clinical data have indicated that PAH-related hospitalizations are associated with adverse clinical outcomes. Data analyses from the REVEAL registry and SERAPHIN and GRIPHON trials have shown associations between mortality and all-cause hospitalization among patients with $\mathrm{PAH}$, as well as hospitalization for $\mathrm{PAH}$ disease progression [11, 12]. This body of evidence has informed the inclusion of hospitalization in risk and prognostic assessment tools 
for $\mathrm{PAH}$, as well as clinical decision-making $[11,13]$. Real-world studies have also documented a high risk of hospitalization and readmission among this patient population [14-16]. Similar studies have confirmed associations between hospitalization and mortality [17-19].

Evaluation of $\mathrm{PAH}$ treatment management surrounding hospitalization may also offer insight into clinical practice. The REVEAL 2.0 survival risk score for PAH includes all-cause hospitalization, and there is some clinical consensus for its consideration in treatment strategies for certain situations $[13,18]$. Thus, hospitalization can be viewed as an indicator of the need to assess therapy escalation options. In addition, while there are some data evaluating treatment patterns and clinical endpoints before and after PAH treatment initiation [20-22], to date there are no real-world studies specifically evaluating treatment patterns before and after initial hospitalization.

To address this gap, the authors undertook this study to examine the PAH patient characteristics and treatment status (monotherapy, combination therapy [double and triple], and no PAH therapy) before and after initial hospitalization, as reflected in a large US-specific commercial data set. The results shed light on the current treatment landscape in clinical practice and can inform future research and decision-making.

\section{METHODS}

\section{Study Design and Data Sources}

This study was a retrospective cohort analysis conducted with data from January 1, 2014, through June 30, 2019. Baseline characteristics were observed during the 6 months prior to hospitalization, and treatment patterns were observed 30 days pre- and 90 days post-hospitalization (Fig. 1). The data set that was used for this present analysis is the US-specific Optum ${ }^{\circledR}$ Clinformatics $^{\circledR}$ Data Mart database, a large, geographically diverse, de-identified commercial database that includes approximately 69 million enrollees from the United States [23]. Raw data included comprehensive demographic, clinical, and treatment information identified using International Classification of Diseases, Ninth and Tenth Revision, Clinical Modification (ICD-9-CM, ICD-10-CM) codes, National Drug Codes (NDCs), and Healthcare Common Procedure Coding System (HCPCS) codes. In addition, analysis was replicated using two other large US claims databases (MarketScan data set JAN2014-JUN2019 and IQVIA data set JAN2014-MAR2019) to validate the results from the study.

\section{Compliance with Ethics Guidelines}

This retrospective database analysis did not involve the collection, use, or transmittal of individual identifiable data. Thus, institutional review board approval to conduct this study was not required [24], as it is considered exempt according to Common Rule Requirements, 45CFR46.101(b)(4): Existing Data and Specimens-No Identifiers. Both the data set itself and the security of the offices where the data are housed meet the requirements of the Health Insurance Portability and Accountability Act of 1996 [24]. Data were accessed with permission from the Optum, Marketscan, and IQVIA databases via data licensing agreements.

\section{Study Population}

Adult patients who had $\geq 1$ pulmonary hypertension (PH)/PAH diagnosis claim (ICD-9-CM: $416.0,416.8$ or ICD-10-CM: I27.0, I27.2, I27.89) in the inpatient setting or $\geq 2 \mathrm{PH} / \mathrm{PAH}$ diagnoses claims $\geq 30$ days apart in the outpatient setting [25] were identified during the study period, from January 1, 2014, through June 30, 2019 (Fig. 1). The first PH/PAH diagnosis claim was defined as the initial PAH diagnosis date.

Patients were required to have a prescription claim for a targeted PAH medication (see Table 1) on or after the initial PAH diagnosis date [25] and an all-cause hospitalization on or after the first observed PAH prescription claim date during the identification period (ID; 01 June 2014 through 30 June 2019). The first observed all-cause hospitalization during the ID period was defined as the index hospitalization. 


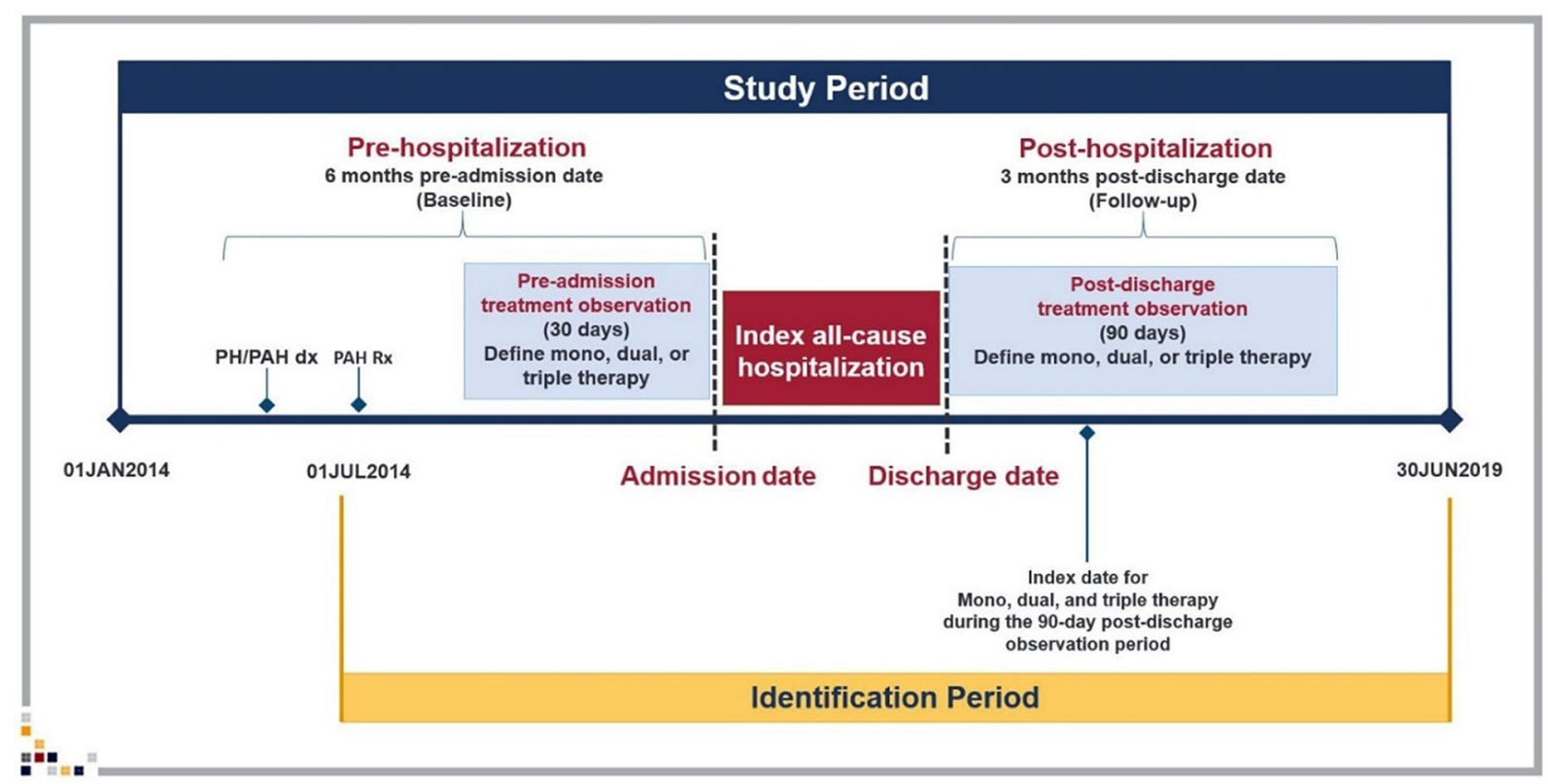

Fig. 1 Study design. $P H$ pulmonary hypertension, $P A H$ pulmonary arterial hypertension, $d x$ diagnosis, $R x$ prescription

Table 1 List of PAH medications

\begin{tabular}{ll}
\hline Category & Medications $^{\mathbf{a}}$ \\
\hline Phosphodiesterase (type 5) enzyme inhibitors (PDE-5i) & Sildenafil \\
Endothelin receptor antagonists (ERAs) & Tadalafil \\
& Macitentan \\
Prostacyclin pathway agents (parenteral) & Ambrisentan \\
& Bosentan \\
Prostacyclin pathway agents (oral) & Iloprost \\
Soluble guanylate cyclase stimulator (SGCS) & Epoprostenol \\
\hline
\end{tabular}

${ }^{a}$ Both National Drug Codes (NDCs) in the pharmacy claims database and Healthcare Common Procedure Coding System (HCPCS) codes in the medical claims database were used to identify PAH medications

Other selection requirements included continuous health plan enrollment for $\geq 6$ months prior to the first all-cause hospitalization (baseline period) and $\geq 3$ months post-discharge (follow-up period). Patients with claims for pregnancy, labor, or erectile dysfunction during the study period were excluded.

Among included patients, flags were created in hierarchical order for evidence of triple therapy, double therapy, monotherapy, or no 
PAH therapy within 30 days before index hospitalization and 90 days after the index hospitalization discharge date.

\section{Study Measures}

\section{Baseline Patient Characteristics}

Patient demographic characteristics were recorded, including sex, age, and geographic region on the index date. In addition, baseline patient clinical characteristics during the 6-month preindex hospitalization period were recorded, including Charlson comorbidity index (CCI) scores [26], individual comorbidities, and specific $\mathrm{PAH}$ treatments during the 6-month period (separate from class-level treatment status 30 days pre-hospitalization).

\section{Study Outcomes}

The primary outcomes were treatment status during the 30-day pre-hospitalization and 90-day post-hospitalization periods among patients with all-cause and PAH-related hospitalizations, and treatment patterns during the same periods among patients with all-cause hospitalizations. Treatment status was defined as follows: monotherapy-patients prescribed a single PAH medication class; double therapypatients prescribed two different classes with $\geq 1$ overlapping days of supply; triple therapypatients prescribed three different classes of PAH medications with $\geq 1$ overlapping day of supply; or No PAH therapy-patients without a refill of the index therapy or with the refill date more than 30 days after the index therapy.

Treatment patterns included modification and continuity in PAH treatment class (no PAH therapy/mono/double/triple) during the 30-day pre-hospitalization and 90-day post-hospitalization periods. Modification events included therapy escalation, de-escalation, and medication switches. Escalation was defined as any increase in the number of specific PAH medications, from no therapy to monotherapy through double therapy, to triple therapy. Deescalation was defined as any decrease in the number of specific PAH medications, from triple to double therapy through monotherapy to no therapy. Medication switches were defined as changes to a different specific medication within a PAH treatment class (i.e., mono/double/triple) without a change in the number of PAH medications. For instance, patients who switched from ERA monotherapy pre-hospitalization to PDE5i monotherapy post-hospitalization are considered a medication switch (Fig. 2).

\section{Statistical Analysis}

All study variables were analyzed descriptively; means, standard deviations, medians, and ranges were reported for continuous variables. Counts and percentages were reported for categorical variables. Analyses were performed using SAS ${ }^{\circledR}$ for Windows, Version 9.4 (SAS Institute, Cary, NC).

\section{RESULTS}

After application of the other selection criteria, a total of 3116 patients with $>1$ all-cause hospitalization was included in the analysis (Fig. 3).

\section{Patient Characteristics}

Demographics at baseline showed that the study population had a mean age of 68.1 years, was predominately female $(68.1 \%)$, and residents of the Southern US region (45.8\%). Clinical characteristics included a mean CCI score of $5.1( \pm 3.0)$. The most prevalent comorbidity was hypertension $(81.5 \%)$, followed by chronic obstructive pulmonary disease (46.9\%). The most prescribed $\mathrm{PAH}$ medications during the 6-month baseline period were PDE-5i (57.8\%), followed by ERAs (29.5\%). The mean all-cause inpatient length of stay for index all-cause hospitalization was $6.6( \pm 8.2)$ days (Table 2$)$.

\section{Study Outcomes}

Overall proportions for treatment status were observed during the 30-day pre- and 90-day post-hospitalization periods among both patients with all-cause hospitalizations and 


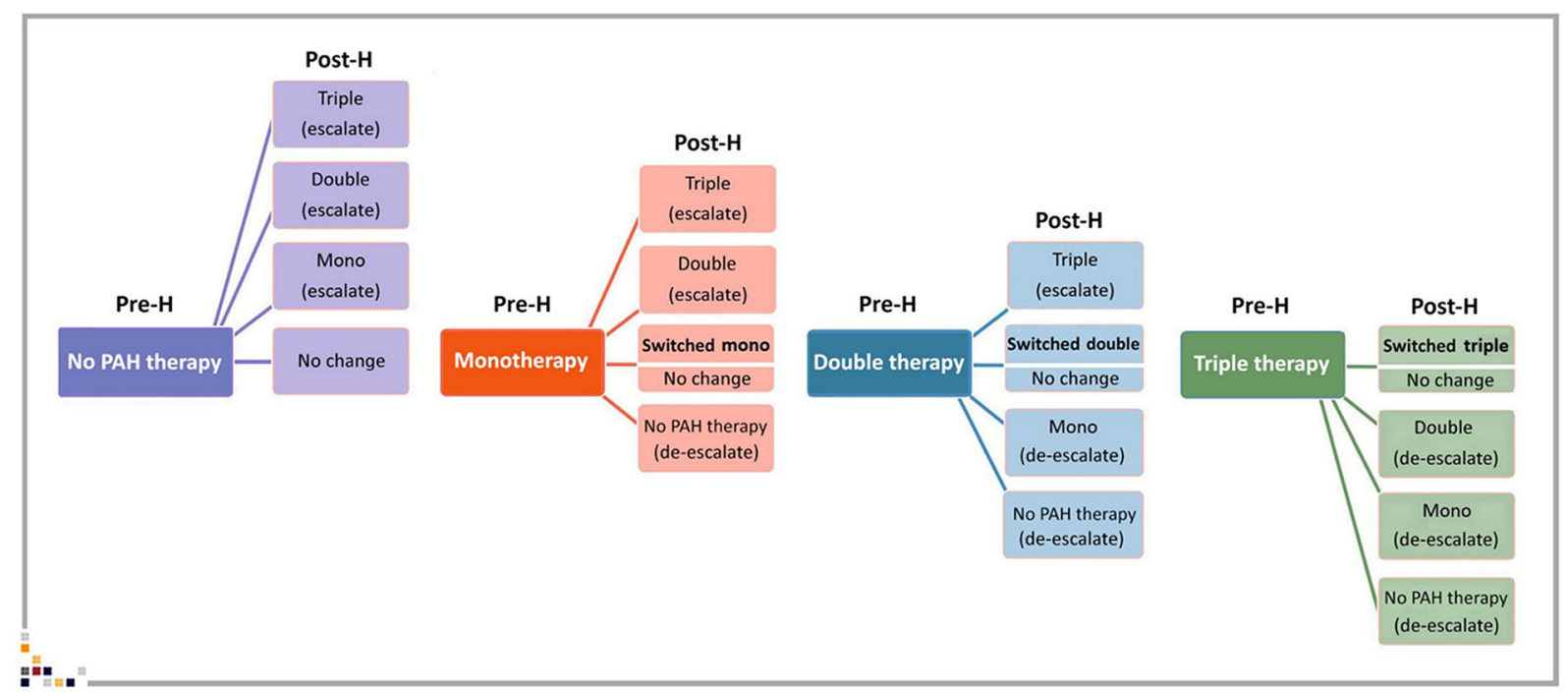

Fig. $2 \mathrm{PAH}$ treatment patterns 30 days pre- and 90 days post-hospitalization. $H$ hospitalization, $P A H$ pulmonary arterial hypertension

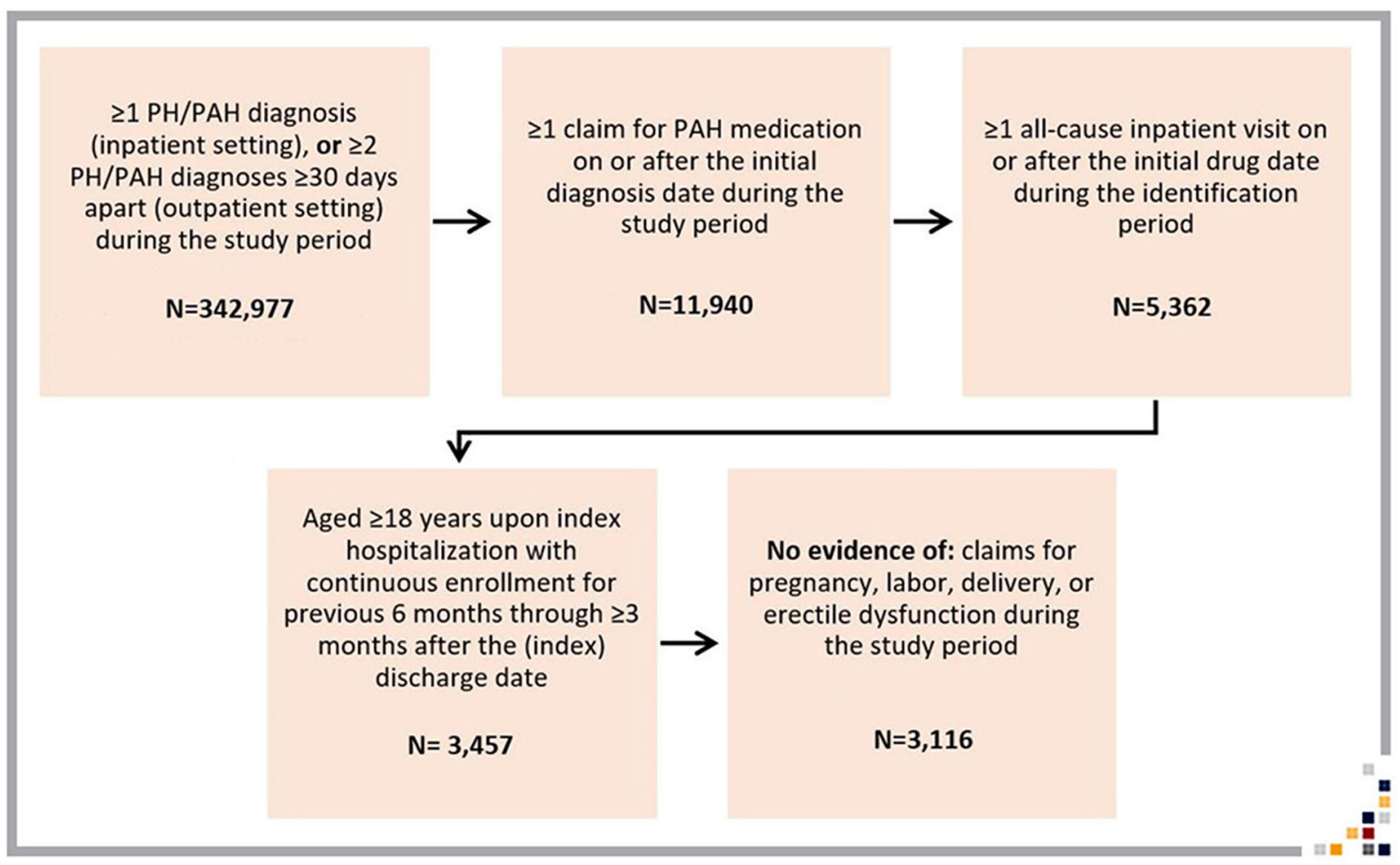

Fig. 3 Patient selection

those with PAH-related hospitalizations. Among the 3116 patients with all-cause hospitalizations, $64.8 \%$ were prescribed monotherapy pre-hospitalization; this reduced to $51.9 \%$ post-hospitalization. Approximately $14.6 \%$ of patients had no PAH therapy 30 days 
Table 2 Descriptive baseline characteristics of hospitalized patients with $\mathrm{PAH}$

\begin{tabular}{|c|c|}
\hline Variables & $\begin{array}{l}\text { Optum } \\
(N=3116) \\
N / \text { Mean }(\% / \\
\text { SD })\end{array}$ \\
\hline Age (years) & $68.1(12.7)$ \\
\hline \multicolumn{2}{|l|}{ Age group (years) } \\
\hline $18-54$ & $438(14.1 \%)$ \\
\hline $55-64$ & $639(20.5 \%)$ \\
\hline$\geq 65$ & $2039(65.4 \%)$ \\
\hline \multicolumn{2}{|l|}{ Sex } \\
\hline Male & $994(31.9 \%)$ \\
\hline Female & $2122(68.1 \%)$ \\
\hline \multicolumn{2}{|l|}{ US geographic region } \\
\hline Northeast & $328(10.5 \%)$ \\
\hline North Central & $751(24.1 \%)$ \\
\hline South & $1428(45.8 \%)$ \\
\hline West/other & $609(19.5 \%)$ \\
\hline \multicolumn{2}{|l|}{ Charlson comorbidity index } \\
\hline Mean CCI & $5.1(3.0)$ \\
\hline \multicolumn{2}{|l|}{ Comorbidities $^{a}$} \\
\hline Hypertension & $2540(81.5 \%)$ \\
\hline Chronic obstructive pulmonary disease & $1461(46.9 \%)$ \\
\hline Diabetes & $1350(43.3 \%)$ \\
\hline $\begin{array}{l}\text { Coronary artery disease/ischemic heart } \\
\text { disease }\end{array}$ & $1271(40.8 \%)$ \\
\hline Renal insufficiency & $1182(37.9 \%)$ \\
\hline Obesity & $819(26.3 \%)$ \\
\hline Lower respiratory disease & $736(23.6 \%)$ \\
\hline Apnea & $580(18.6 \%)$ \\
\hline Depression & $547(17.6 \%)$ \\
\hline Pneumonia & $498(16.0 \%)$ \\
\hline Interstitial lung disease & $479(15.4 \%)$ \\
\hline Sleep disorders & $372(11.9 \%)$ \\
\hline Venous thromboembolism & $338(10.8 \%)$ \\
\hline
\end{tabular}

Table 2 continued

\begin{tabular}{|c|c|}
\hline Variables & $\begin{array}{l}\text { Optum } \\
(N=3116) \\
N / \text { Mean }(\% / \\
\text { SD) }\end{array}$ \\
\hline Liver disease & $306(9.8 \%)$ \\
\hline Congenital heart disease & $211(6.8 \%)$ \\
\hline Idiopathic pulmonary fibrosis & $134(4.3 \%)$ \\
\hline Left heart failure & $108(3.5 \%)$ \\
\hline \multicolumn{2}{|l|}{ Baseline PAH medications ${ }^{\mathrm{a}}$} \\
\hline $\begin{array}{l}\text { Phosphodiesterase (type 5) enzyme } \\
\text { inhibitors (PDE-5i) }\end{array}$ & $1801(57.8 \%)$ \\
\hline Endothelin receptor antagonists (ERAs) & $920(29.5 \%)$ \\
\hline Prostacyclin pathway agents & $337(10.8 \%)$ \\
\hline $\begin{array}{l}\text { Soluble guanylate cyclase stimulator } \\
(\mathrm{sGCS})\end{array}$ & $191(6.1 \%)$ \\
\hline \multicolumn{2}{|l|}{ All-cause index hospitalization } \\
\hline Length of stay (days) & $6.6(8.2)$ \\
\hline
\end{tabular}

pre-hospitalization and $24.5 \%$ had no $\mathrm{PAH}$ therapy 90 days post-hospitalization. Approximately $17.4 \%$ and $3.2 \%$ of the patients were prescribed double therapy and triple therapy, respectively, during the 30-day pre-hospitalization period. There was a slight decrease in the proportions post-hospitalization (double therapy [16.5\%], triple therapy [3.1\%]) (Fig. 4a).

Of the total hospitalized patient population, $34.1 \%(n=1062)$ had a PAH-related hospitalization. Among these patients, 60.8\% were prescribed monotherapy pre-hospitalization, which reduced to $49.1 \%$ post-hospitalization. The next most-common post-hospitalization status was "no evidence of therapy", which increased from 10.0\% 30-day pre-hospitalization to $22.8 \%$ 90-day post-hospitalization. Combined patients prescribed double $(23.5 \%)$ and triple therapy $(5.7 \%)$ also accounted for about a third of the total during 30-day prehospitalization, with generally stable proportions from pre- to post-hospitalization (double 
therapy [22.4\%] and triple therapy [5.7\%]) (Fig. 4b).

Among the 3116 patients with all-cause hospitalizations, about $20.0 \%$ had therapy deescalation, $6.1 \%$ had therapy escalation, and $72.8 \%$ had no change in treatment (Table 3).

Among the 2020 patients prescribed monotherapy 30 days pre-hospitalization, the majority $(72.0 \%)$ remained on monotherapy in the 90-day post-hospitalization period; of these, 98.0\% remained on the same monotherapy. Approximately $23.1 \%$ of patients with pre-hospitalization monotherapy had no PAH therapy post-hospitalization (Fig. 5).

Among the 541 patients prescribed double therapy 30 days pre-hospitalization, the majority $(72.5 \%)$ remained on double therapy in the 90-day post-hospitalization period $(99.0 \%$ same double therapy); de-escalation to monotherapy $(17.7 \%)$ was the next most common outcome (Fig. 5).

Among the 98 patients prescribed triple therapy 30 days pre-hospitalization, the majority $(69.4 \%)$ remained on triple therapy in the 90 -day post-hospitalization period (100\% same triple therapy); de-escalation to double therapy $(22.4 \%)$ was the next most common outcome (Fig. 5).

Among the 456 patients with no evidence of PAH therapy during the 30 days pre-hospitalization, the majority $(84.6 \%)$ remained without $\mathrm{PAH}$ therapy in the 90-day post-hospitalization period; escalation to monotherapy (14.3\%) was the next most common outcome (Fig. 5).

No PAH therapy during the 30 days prehospitalization was defined by the absence of any claim for PAH-specific medication. No PAH therapy during the 90 days post-hospitalization was defined by the absence of any claim for PAH-specific medication or a 30-day claim without subsequent refill.

\section{DISCUSSION}

This study evaluated key patient characteristics and treatment patterns among patients with $\mathrm{PAH}$ who were enrolled in a large US commercial claims database. The results provide insight into hospitalization as a critical juncture in the
PAH patient journey, and its role in disease management in clinical practice. Results showed high comorbidity among hospitalized patients with PAH (including both PAH-related and all-cause hospitalizations), a predominance of monotherapy throughout the treatment observation period, and high proportions of patients without PAH therapy. Among analyzed patients with all-cause hospitalizations, the overwhelming majority did not have any treatment modification within 90 days of discharge from their index hospitalization. Of those with modification, most de-escalated. These results suggest that hospitalization per se did not have a major impact on PAH therapy management, which indicates that some providers may not be considering hospitalization as a marker of disease progression that warrants treatment intensification. Moreover, the predominance of monotherapy raises questions about discrepancies with clinical guideline indications for combination therapy.

One point of note is that while we stratified overall proportions of treatment classes before and after hospitalization for both all-cause and PAH-related hospitalizations, this study focused exclusively on all-cause hospitalizations to observe post-hospitalization treatment modification. This is in part because observation of overall proportions followed closely similar trends between patients with all-cause and PAHrelated hospitalizations in the present data set. These trends suggest that PAH-related admissions drove all-cause hospitalizations in this study population with high comorbidity, which generally aligns with a previous study of firsttime hospitalizations among patients newly diagnosed with PAH in the REVEAL registry [14]. In that study, Burger et al. hypothesize that the distinction between PAH-related and other hospitalizations may be arbitrary among patients with $\mathrm{PAH}$, as admissions attributed to comorbidities are likely to have been exacerbated by the PAH [14]. There is also precedent for this methodological approach in a study of the REVEAL 2.0 algorithm and European Society of Cardiology/European Respiratory Society (ESC/ERS) risk assessment guidelines, as the REVEAL 2.0 algorithm includes only all-cause hospitalization, and many admissions are coded 
A

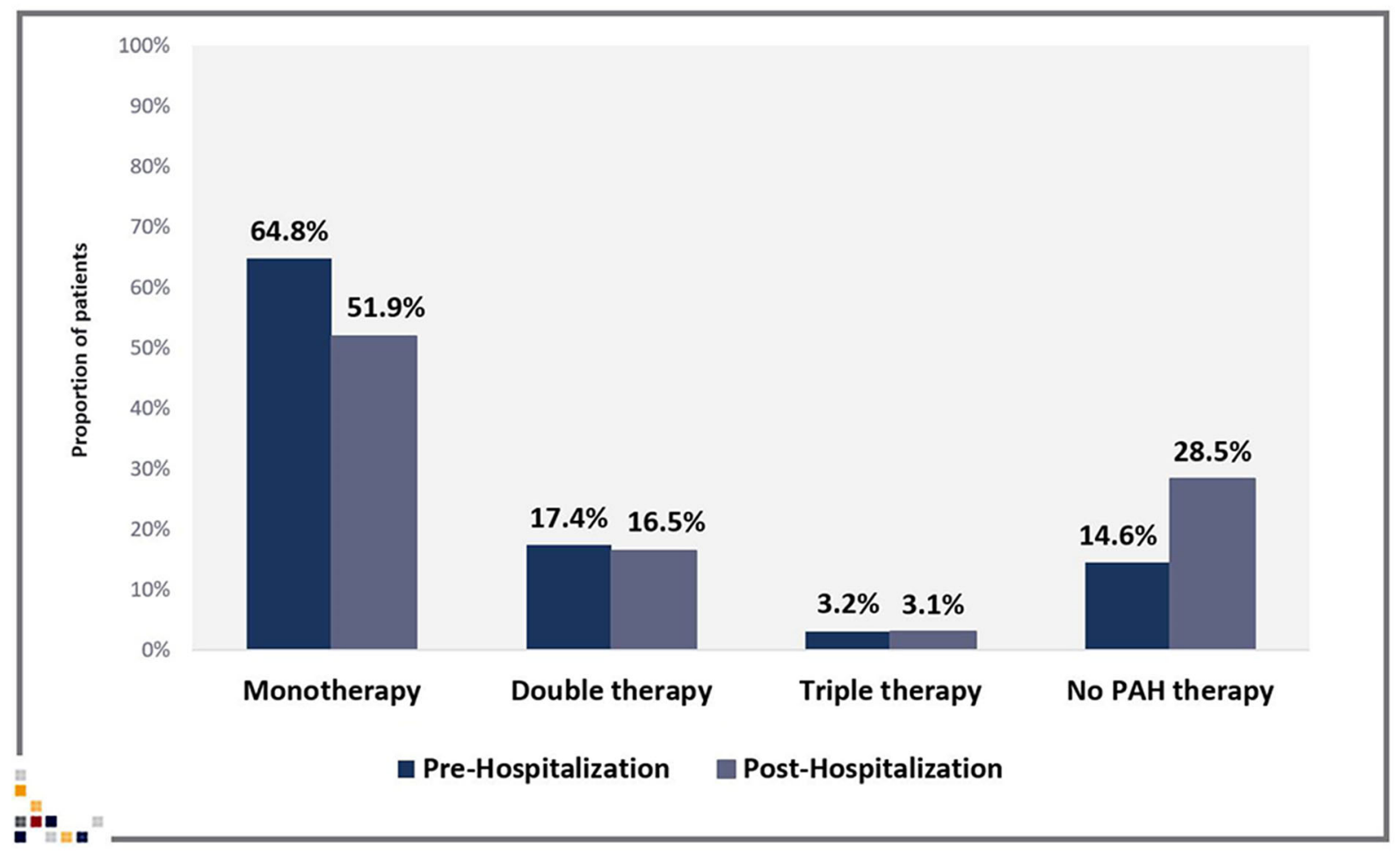

B

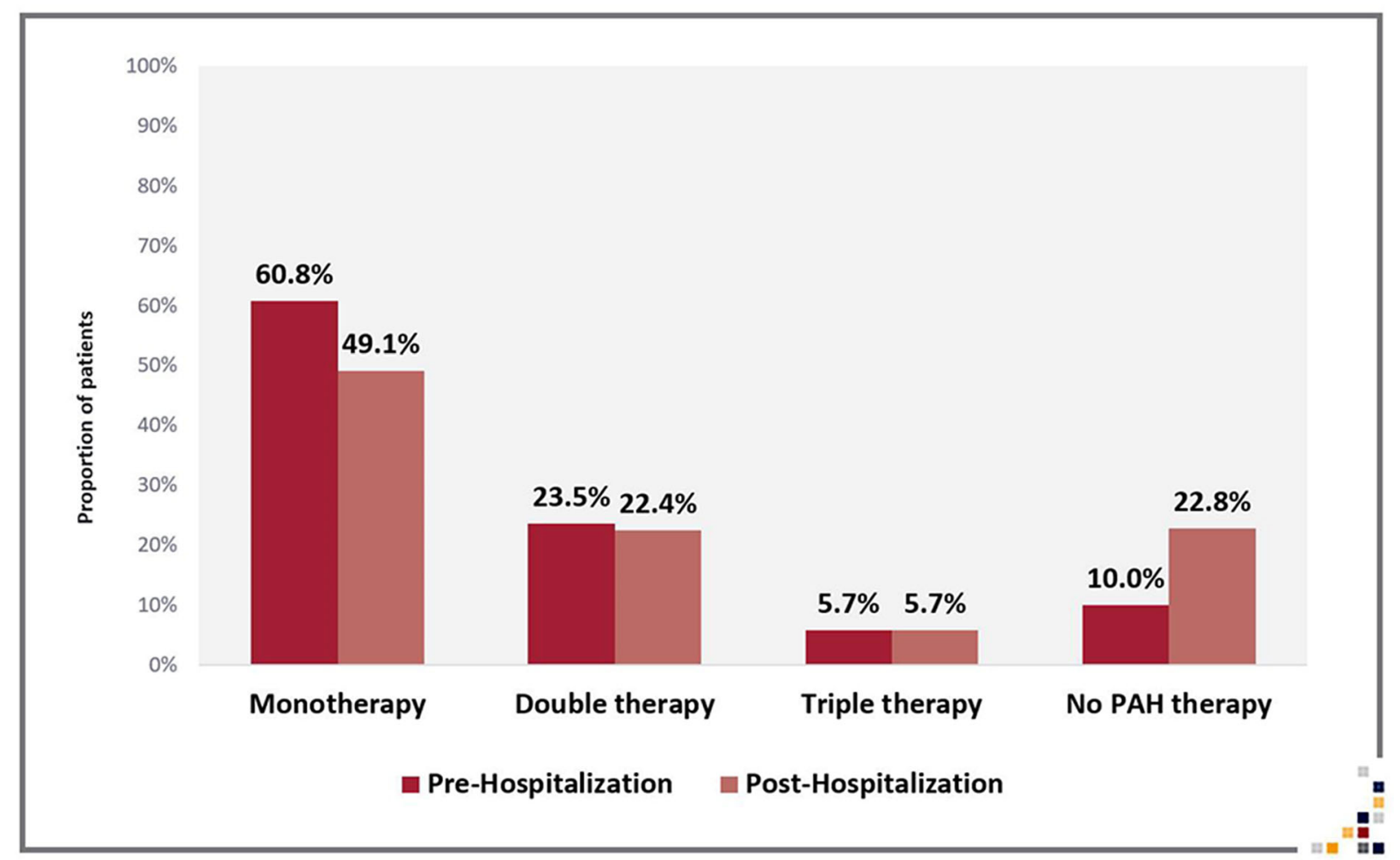


4Fig. 4 a Treatment status pre- and post-all cause hospitalization among hospitalized PAH patients. b Treatment status pre- and post-PAH-related hospitalization among hospitalized PAH patients. No PAH therapy during the 30 days pre-hospitalization was defined by the absence of any claim for PAH-specific medication. No PAH therapy during the 90 days post-hospitalization was defined by the absence of any claim for PAH-specific medication or a 30-day claim without subsequent refill

for other conditions that are in fact PAH-related comorbidities [27]

Also of note, we replicated the analyses presented here in two additional large commercial data sets to validate the results (MarketScan: $\mathrm{N}=2034$; IQVIA: $N=1403$ ). The specific results and general trends were closely similar in both ad hoc analyses. Selected results appear in Supplementary Tables 1 and 2.

The predominance of monotherapy observed in this study aligns with previous studies, although the proportions of patients prescribed combination therapy trends higher in this study $[22,29,30]$. In addition, the results add to evidence suggesting a slow but steady shift toward greater use of combination therapy that generally coincides with the introduction of pivotal clinical guidelines in 2015 (ESC/ERS pulmonary hypertension guidelines). Specifically, real-world data show that the proportions of patients with $\mathrm{PAH}$ prescribed combination therapy increased from $5 \%$ before the release of the abovementioned guidelines to $\sim 20 \%$ observed in the present study (with most patients [72.2\%] observed after 2015) [22, 28].

Nonetheless, while combination therapy appears to be gaining traction, the data still appear to speak to a persisting lag between evidence favoring greater and earlier use of combination therapy in general $[9,10,28-32]$. In this context, the low levels of treatment escalation we observed after hospitalization suggest that many clinicians are not making a full connection between hospitalization, clinical worsening, and the need for treatment intensification. We would expect otherwise, given that studies have shown combination therapy to be associated with better outcomes among patients with $\mathrm{PAH}$; for example, combination-treated patients have substantial reductions in clinical worsening relative to those treated with monotherapy [33]. The importance of hospitalization for survival prognosis and prediction of future hospitalization is also well established in the literature. [12, 13, 16-19, 34]. However, to our knowledge, the present study results regarding the coincidence of treatment modification and hospitalization are novel. While continued research is necessary, these exploratory results suggest there may be a gap between guidelines and clinical practice that should be closed.

Regardless, the results warrant continued research to test these results and better understand the reasons for limited escalation and other modification following hospitalization that may be playing out in routine practice. One possible explanation is that some providers may

Table 3 Treatment modification pre- and post-all cause hospitalization

\begin{tabular}{|c|c|c|c|c|}
\hline & \multicolumn{4}{|c|}{ Post-hospitalization modification } \\
\hline & No change & Medication switch & Escalation & De-escalation \\
\hline Pre-hospitalization treatment status ${ }^{a}$ & $N=2267(72.8 \%)$ & $N=33(1.0 \%)$ & $N=191(6.1 \%)$ & $N=624(20.0 \%)$ \\
\hline Monotherapy & $125(45.7 \%)$ & $29(0.9 \%)$ & $99(3.2 \%)$ & $467(15.0 \%)$ \\
\hline Double therapy & $388(12.5 \%)$ & $4(0.1 \%)$ & $22(0.7 \%)$ & $127(4.0 \%)$ \\
\hline Triple therapy & $68(2.2 \%)$ & - & - & $30(1.0 \%)$ \\
\hline No PAH therapy & $386(12.4 \%)$ & - & $70(2.2 \%)$ & - \\
\hline
\end{tabular}

${ }^{a}$ Percentages do not equal $100 \%$ due to rounding 


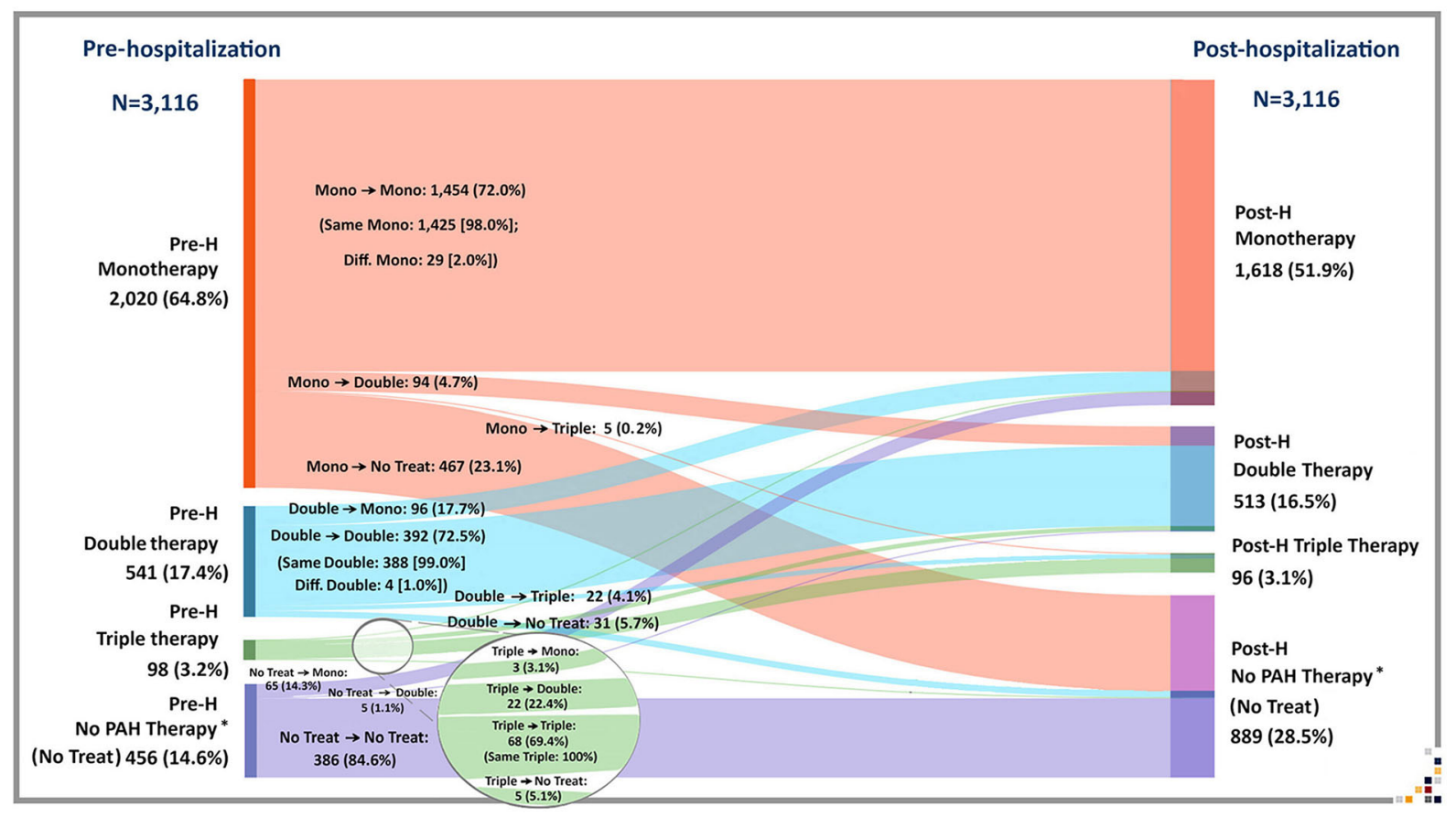

Fig. 5 Treatment status patterns pre- and post-all cause hospitalization. ${ }^{*}$ No PAH therapy (No Treat): No PAH therapy during the 30 days pre-hospitalization was defined by the absence of any claim for PAH-specific medication.
No PAH therapy during the 90 days post-hospitalization was defined by the absence of any claim for PAH-specific medication or a 30-day claim without subsequent refill lack access to hospitalization data when they must make treatment decisions, which can be urgent among this patient population [13]. Another factor may be clinicians who primarily inform their decision-making with current guidelines that neither include hospitalization as a specific risk factor for $\mathrm{PAH}$ progression nor specifically call for modification upon hospitalization $[1,10]$. In addition, while the REVEAL 2.0 risk algorithm does include hospitalization as a variable, other algorithms do not, and the proportion of clinicians who rely primarily on guidelines and non-REVEAL risk profiles is currently unknown. Comorbidity may also cause reluctance among clinicians to initiate combination therapy, and contraindications among concomitant medications as well as polypharmacy per se may also curtail treatment choices $[29,35,36]$. Thus, the considerable comorbidity we observed is notable, especially given that several of the observed specific PAH medications have contraindications for comorbidities prevalent in the sample (e.g., hypertension, renal dysfunction, liver disorders, chronic obstructive pulmonary disease) [37-44]. Lastly, tolerability issues that cannot be observed in claims data may have curtailed treatment options and driven some of the de-escalation we observed.

While continued research may be necessary to validate the present study results and gain sufficient insight into clinical decision-making, ultimately a case may be made for adding more explicit connections between hospitalization and treatment intensification to existing guidelines, and for more uniform consideration of hospitalization in risk assessment.

The results of this study also suggest value in future real-world research on the reasons for treatment continuity and de-escalation, as well as the effects of both de-escalation and guideline-recommended escalation on patient outcomes, re-admission, and costs. Such research would also benefit from analyses across 
treatment classes and specific medications, as well as among study populations stratified by comorbidity and concomitant medication use. More granular investigations may help clinicians accurately identify and effectively treat patients with PAH who would benefit from treatment escalation.

\section{Strengths and Limitations}

A key strength of this study design is the large sample of patients with $\mathrm{PAH}$, who remain understudied in clinical practice. In addition, the consistency of the results across databases reinforces the study power and robustness of the methods, and the novelty of retrospective evaluation of treatment status before and after hospitalization offers insight into a critical juncture for which there is currently little realworld evidence. This insight into the PAH treatment landscape and patient journey provides fertile ground for future hypothesis generation.

However, the study also has limitations. As with all retrospective designs, the present study is limited to the observation of associations as opposed to inference of causality. Moreover, while claims data are extremely valuable to augment randomized controlled trial results with insight into clinical practice, all claim databases have certain inherent limitations because the claims are collected for administrative purposes and not research, and thus may lack certain clinical information. The present study was specifically limited in that certain clinical information was not observable in the data set, such as etiology, World Health Organization functional class, risk assessment, hemodynamics, vasoreactivity, and other clinical parameters. Similarly, claims data cannot capture clinical reasoning regarding comorbid conditions, medication contraindications, and tolerability issues. There is no specific ICD-9CM or ICD-10-CM code for PAH, and it is conceivable that an unknown proportion of patients in this study may have other forms of $\mathrm{PH}$. Furthermore, the presence of a diagnosis for any disease on medical claims is not clinically validated, as the diagnosis code may be incorrectly coded or included as rule-out criteria rather than the actual disease.

In addition, while identification of $\mathrm{PAH}$ diagnoses followed an algorithm in line with validated precedents $[16,25]$, it relied on diagnosis codes that may have been subject to coding discrepancies. Also, riociguat is indicated for both PAH and chronic thromboembolic pulmonary hypertension (CTEPH) patients, so there is a possibility of misclassification. As with most claims data sources, inpatient pharmacy claims were not observable. However, the relatively short hospitalization period (mean length of stay [LOS] of 7 days) was unlikely to deviate from the trends that were consistent from the pre- through the post-discharge periods.

\section{CONCLUSIONS}

This large, retrospective real-world analysis of hospitalized patients with $\mathrm{PAH}$ found patients to have high comorbidity levels, a predominance of prescribed monotherapy throughout the evaluation period (pre- and post-hospitalization), and a majority with no treatment escalation or other modification despite hospitalization. Among those prescribed monotherapy 30 days pre-hospitalization who did have modification within 90 days post-hospitalization, de-escalation to no therapy was most common, accounting for nearly a quarter of this subgroup. These results suggest a persistent gap between real-world clinical practice and the evidence on the benefits of therapy in general and guidelines asserting the need for combination therapy. The results warrant continued research into the reasons for limited treatment modification as well as the impact of post-hospitalization optimization on clinical and economic outcomes such as progression, readmission, death, and total cost of care. Ultimately, such research may point to a need for hospitalization and subsequent therapeutic management as a quality measure across health care systems. Real-world clinical practice may be positively influenced by the consideration of hospitalization and recommendations for therapy management by expert guidelines and risk assessment algorithms. The data from this study 
demonstrate a need for improved treatment decision-making for this vulnerable patient population.

\section{ACKNOWLEDGEMENTS}

Funding. Sponsorship for this study as well as all publication charges were funded by Janssen Scientific Affairs.

Medical Writing, Editorial, or Other Assistance. Medical writing assistance was provided by Michael Kane of STATinMED Research; this assistance was funded by the study sponsors.

Authorship. All named authors meet the International Committee of Medical Journal Editors (ICMJE) criteria for authorship for this article, take responsibility for the integrity of the work as a whole, and have given their approval for this version to be published.

Author Contributions. AO, YT, FK, WLT, and SP contributed to the study development and design. AO, YT, FK, WLT, and SP were involved in the acquisition of data, analysis, and interpretation of results. AO, YT, FK, WLT, and SP were involved in all stages of manuscript development, writing and revision.

Prior Presentation. Data from this study have been previously presented in poster format at the Academy of Managed Care Pharmacy (AMCP) Conference.

Disclosures. Yuen Tsang and Sumeet Panjabi are employees of Janssen Scientific Affairs, LLC, a Janssen Pharmaceutical Company of Johnson \& Johnson in Titusville, NJ, the study sponsor. Adesuwa Ogbomo and Wan-Lun Tsai are employees of STATinMED Research, a paid consultant to the study sponsors, as was Furaha Kariburyo at the time of the study.

Compliance with Ethics Guidelines. This retrospective database analysis did not involve the collection, use, or transmittal of individual identifiable data. Thus, institutional review board approval to conduct this study was not required [24], as it is considered exempt according to Common Rule Requirements, 45CFR46.101(b)(4): Existing Data and Specimens-No Identifiers. Both the data set itself and the security of the offices where the data are housed meet the requirements of the Health Insurance Portability and Accountability Act of 1996. Data were accessed with permission from the Optum, Marketscan, and IQVIA databases via data licensing agreements.

Data Availability. The authors had full permission from three commercial data sources to access the data sets and use them for this study. However, restrictions apply to the dissemination of these data, which were used under license for the current study on; thus, these data are not publicly available.

Open Access. This article is licensed under a Creative Commons Attribution-NonCommercial 4.0 International License, which permits any non-commercial use, sharing, adaptation, distribution and reproduction in any medium or format, as long as you give appropriate credit to the original author(s) and the source, provide a link to the Creative Commons licence, and indicate if changes were made. The images or other third party material in this article are included in the article's Creative Commons licence, unless indicated otherwise in a credit line to the material. If material is not included in the article's Creative Commons licence and your intended use is not permitted by statutory regulation or exceeds the permitted use, you will need to obtain permission directly from the copyright holder. To view a copy of this licence, visit http://creativecommons.org/licenses/bync/4.0/.

\section{REFERENCES}

1. Galiè N, Humbert M, Vachiery JL, et al. 2015 ESC/ ERS Guidelines for the diagnosis and treatment of pulmonary hypertension: the Joint Task Force for the Diagnosis and Treatment of Pulmonary Hypertension of the European Society of Cardiology (ESC) and the European Respiratory Society (ERS). Eur 
Heart J. 2016;37(1):67-119. https://doi.org/10. 1093/eurheartj/ehv317.

2. National Organization for Rare Disorders. Pulmonary arterial hypertension. https://rarediseases. org/rare-diseases/pulmonary-arterial-hypertension/ \#: :text=New\%20cases $\% 20$ are $\% 20$ estimated $\%$ 20to,frequency $\% 20$ of $\% 20$ patients $\% 20$ with $\%$ 20PAH. Accessed 2 Feb 2021.

3. Farber HW, Miller DP, Poms AD, et al. Five-year outcomes of patients enrolled in the REVEAL Registry. Chest. 2015;148(4):1043-54. https://doi.org/ 10.1378/chest.15-0300.

4. McGoon MD, Miller DP. REVEAL: a contemporary US pulmonary arterial hypertension registry. Eur Respir Rev. 2012;21(123):8-18. https://doi.org/10. $1183 / 09059180.00008211$.

5. Leber L, Beaudet A, Muller A. Epidemiology of pulmonary arterial hypertension and chronic thromboembolic pulmonary hypertension: identification of the most accurate estimates from a systematic literature review. Pulm Circ. 2021;11(1): 2045894020977300. https://doi.org/10.1177/ 2045894020977300.

6. $\mathrm{Gu} \mathrm{S}, \mathrm{Hu} \mathrm{H}$, Dong H. Systematic review of the economic burden of pulmonary arterial hypertension. Pharmacoeconomics. 2016;34(6):533-50. https://doi.org/10.1007/s40273-015-0361-0.

7. Maron BA, Galiè N. Diagnosis, treatment, and clinical management of pulmonary arterial hypertension in the contemporary era: a review. JAMA Cardiol. 2016;1(9):1056-65. https://doi.org/10. 1001/jamacardio.2016.4471.

8. Ghofrani HA, Humbert M. The role of combination therapy in managing pulmonary arterial hypertension. Eur Respir Rev. 2014;23(134):469-75. https:// doi.org/10.1183/09059180.00007314 (Epub 2014 Dec 1 PMID: 25445945).

9. Galiè N, Barberà JA, Frost $\mathrm{AE}$, et al. Initial use of ambrisentan plus tadalafil in pulmonary arterial hypertension. N Engl J Med. 2015;373(9):834-44. https://doi.org/10.1056/NEJMoa1413687.

10. Klinger JR, Elliott CG, Levine DJ, et al. Therapy for pulmonary arterial hypertension in adults: update of the CHEST guideline and expert panel report. Chest. 2019;155(3):565-86. https://doi.org/10. 1016/j.chest.2018.11.030 (Epub 2019 Jan 17. Erratum in: Chest. 2021 Jan;159(1):457. PMID: 30660783).

11. Kanwar M, Raina A, Lohmueller L, Kraisangka J, Benza R. The use of risk assessment tools and prognostic scores in managing patients with pulmonary arterial hypertension. Curr Hypertens Rep.
2019;21(6):45. https://doi.org/10.1007/s11906-0190950-y.

12. McLaughlin VV, Hoeper MM, Channick RN, et al. Pulmonary arterial hypertension-related morbidity is prognostic for mortality. J Am Coll Cardiol. 2018;71(7):752-63. https://doi.org/10.1016/j.jacc. 2017.12.010.

13. McLaughlin VV, Channick R, De Marco T, et al. Results of an expert consensus survey on the treatment of pulmonary arterial hypertension with oral prostacyclin pathway agents. Chest. 2020;157(4): 955-65. https://doi.org/10.1016/j.chest.2019.10. 043.

14. Burger CD, Long PK, Shah MR, et al. Characterization of first-time hospitalizations in patients with newly diagnosed pulmonary arterial hypertension in the REVEAL registry. Chest. 2014;146(5): 1263-73. https://doi.org/10.1378/chest.14-0193.

15. Frantz RP, Schilz RJ, Chakinala MM, et al. Hospitalization and survival in patients using epoprostenol for injection in the PROSPECT observational study. Chest. 2015;147(2):484-94. https://doi.org/ 10.1378/chest.14-1004.

16. Burke JP, Hunsche E, Regulier E, Nagao M, Buzinec $\mathrm{P}$, Drake IW. Characterizing pulmonary hypertension-related hospitalization costs among Medicare Advantage or commercially insured patients with pulmonary arterial hypertension: a retrospective database study. Am J Manag Care. 2015;21(3 Suppl):s47-58.

17. Kim N, Farber HW, Highland K, et al. PCV3 hospitalization rates and association with survival risk at baseline in patients with pulmonary artery hypertension (PAH) receiving selexipag in real-world (RW) clinical practice. Value Health. 2020;23:S486. https://doi.org/10.1016/j.jval.2020.08.494.

18. Haddad F, Peterson T, Fuh E, et al. Characteristics and outcome after hospitalization for acute right heart failure in patients with pulmonary arterial hypertension. Circ Heart Fail. 2011;4(6):692-9. https://doi.org/10.1161/CIRCHEARTFAILURE.110. 949933 (Epub 2011 Sep 9 PMID: 21908586).

19. Burger CD, Ghandour M, Padmanabhan Menon D, Helmi H, Benza RL. Early intervention in the management of pulmonary arterial hypertension: clinical and economic outcomes. Clinicoecon Outcomes Res. 2017;24(9):731-9. https://doi.org/ 10.2147/CEOR.S119117.PMID:29200882;PMCID: PMC5703162.

20. White RJ, Vonk-Noordegraaf A, Rosenkranz S, et al. Clinical outcomes stratified by baseline functional class after initial combination therapy for 
pulmonary arterial hypertension. Resp Res. 2019;20(1):208. https://doi.org/10.1186/s12931019-1180-1.

21. Sikirica M, Iorga SR, Bancroft T, Potash J. The economic burden of pulmonary arterial hypertension (PAH) in the US on payers and patients. BMC Health Serv Res. 2014;14(1):676. https://doi.org/10. 1186/s12913-014-0676-0.

22. Burger CD, Ozbay AB, Lazarus HM, et al. Treatment patterns and associated health care costs before and after treatment initiation among pulmonary arterial hypertension patients in the United States. J Manag Care Spec Pharm. 2018;24(8):834-42. https://doi.org/10.18553/jmcp.2018.17391.

23. Sahay S, Tsang Y, Flynn M, Agron P, Dufour R. Burden of pulmonary hypertension in patients with portal hypertension in the United States: a retrospective database study. Pulm Circ. 2020;10(4): $2045894020962917 . \quad$ https://doi.org/10.1177/ 2045894020962917.

24. Berger A, Edelsberg J, Teal S, et al. Changes in healthcare utilization and costs associated with sildenafil therapy for pulmonary arterial hypertension: a retrospective cohort study. BMC Pulm Med. 2012;12:75. https://doi.org/10.1186/1471-2466-1275 .

25. Mathai SC, Hemnes AR, Manaker S, et al. Identifying patients with pulmonary arterial hypertension using administrative claims algorithms. Ann Am Thorac Soc. 2019;16(7):797-806. https://doi.org/10. 1513/AnnalsATS.201810-672CME.

26. Frenkel WJ, Jongerius EJ, Mandjes-van Uitert MJ, van Munster BC, de Rooij SE. Validation of the Charlson Comorbidity Index in acutely hospitalized elderly adults: a prospective cohort study. J Am Geriatr Soc. 2014;62(2):342-6. https://doi.org/10. $1111 /$ jgs. 12635.

27. Benza RL, Miller DP, Gomberg-Maitland M, et al. Predicting survival in pulmonary arterial hypertension: insights from the Registry to Evaluate Early and Long-Term Pulmonary Arterial Hypertension Disease Management (REVEAL). Circulation. 2010;122(2):164-72. https://doi.org/10.1161/ CIRCULATIONAHA.109.898122.

28. Studer S, Hull M, Pruett J, Elliott C, Tsang Y, Drake W. Retrospective database analysis of treatment patterns among patients with pulmonary arterial hypertension. Pulm Ther. 2020;6(1):79-92. https:// doi.org/10.1007/s41030-019-00106-4.

29. Hoeper MM, Kramer T, Pan Z, et al. Mortality in pulmonary arterial hypertension: prediction by the 2015 European pulmonary hypertension guidelines risk stratification model. Eur Respir J. 2017;50(2):
1700740. https://doi.org/10.1183/13993003.007402017.

30. Pulido T, Adzerikho I, Channick RN, et al. Macitentan and morbidity and mortality in pulmonary arterial hypertension. N Engl J Med. 2013;369(9): 809-18. https://doi.org/10.1056/NEJMoa1213917.

31. Sitbon O, Janis X, Savale L, et al. Upfront triple combination therapy in pulmonary arterial hypertension: a pilot study. Eur Respir J. 2014;43:1691-7. https://doi.org/10.1183/09031936.00116313.

32. Lajoie AC, Lauziere G, Leg JC, et al. Combination therapy versus monotherapy for pulmonary arterial hypertension: a meta-analysis. Lancet Respir Med. 2016;4:291-305. https://doi.org/10.1016/S22132600(16)00027-8.

33. Lajoie AC, Lauzière G, Lega JC, et al. Combination therapy versus monotherapy for pulmonary arterial hypertension: a meta-analysis. Lancet Respir Med. 2016;4(4):291-305. https://doi.org/10.1016/S22132600(16)00027-8 (Epub 2016 Feb 27. Erratum in: Lancet Respir Med. 2016 Jun;4(6):e34. PMID: 26935844).

34. Dufour R, Pruett J, Hu N, et al. Healthcare resource utilization and costs for patients with pulmonary arterial hypertension: Real-world documentation of functional class. J Med Econ. 2017;20(11):1178-86. https://doi.org/10.1080/13696998.2017.1363049.

35. Lang IM, Palazzini M. The burden of comorbidities in pulmonary arterial hypertension. Eur Heart J Suppl. 2019;21(Suppl K):K21-8. https://doi.org/10. 1093/eurheartj/suz205.

36. Xanthouli P, Koegler M, Marra AM, et al. Risk stratification and prognostic factors in patients with pulmonary arterial hypertension and comorbidities a cross-sectional cohort study with survival follow-up. Respir Res. 2020;21(1):127. https://doi. org/10.1186/s12931-020-01393-1.

37. U.S. Food \& Drug Administration label: sildenafil. https://www.accessdata.fda.gov/drugsatfda_docs/ label/2014/20895s039s042lbl.pdf. Accessed 28 Apr 2021.

38. U.S. Food \& Drug Administration label: tadalafil. https://www.accessdata.fda.gov/drugsatfda_docs/ label/2011/021368s20s21lbl.pdf. Accessed 28 Apr 2021.

39. U.S. Food \& Drug Administration label: macitentan. Available at: https://www.accessdata.fda.gov/ drugsatfda_docs/label/2019/204410s017lbl.pdf. Accessed 28 Apr 2021.

40. U.S. Food \& Drug Administration label: ambrisentan. https://www.accessdata.fda.gov/drugsatfda 
docs/label/2015/022081s033lbl.pdf. Accessed 28 Apr 2021.

41. U.S. Food \& Drug Administration label: bosentan. https://www.accessdata.fda.gov/drugsatfda_docs/ label/2009/021290s012lbl.pdf. Accessed 28 Apr 2021.

42. U.S. Food \& Drug Administration label: iloprost. https://www.accessdata.fda.gov/drugsatfda_docs/ label/2009/021779s009lbl.pdf. Accessed 28 Apr 2021.
43. U.S. Food \& Drug Administration label: selexipag. https://www.accessdata.fda.gov/drugsatfda_docs/ label/2015/207947s000lbl.pdf. Accessed 28 Apr 2021.

44. U.S. Food \& Drug Administration label: treprostinil. https://www.accessdata.fda.gov/drugsatfda_docs/ label/2013/021272s020lbledt.pdf. Accessed 28 Apr 2021. 